\author{
Military Technical College \\ Kobry El-Kobba, \\ Cairo, Egypt
}

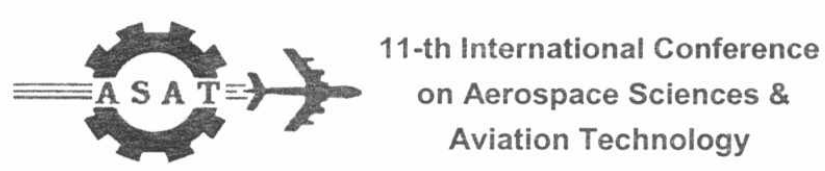

Aviation Technology

\title{
PREDICTION AND CONTROL OF FLOW ASYMMETRY OVER SLENDER CONICAL BODIES.
}

Hamdy. A. Kandil ${ }^{11}$, Hassan. A. Warda ${ }^{1}$, Alaa. A. El-Meligui ${ }^{1}$, Wael. A. Azab ${ }^{1}$.

\section{ABSTRACT}

The supersonic locally-conical flow over a $5^{\circ}$ semi-apex circular cone at a Mach number of 1.8 and angles of attack (AOA) ranging from $10^{\circ}$ to $35^{\circ}$ is studied computationally using the three-dimensional Navier-Stokes equations. An implicit upwind flux-difference splitting scheme was used to obtain the numerical solution of the governing equations. Three flow patterns were predicted depending on the $A O A$. These flow patterns are; steady symmetric flow at $A O A=10^{\circ}$, steady asymmetric flow for $15^{\circ} \leq \mathrm{AOA} \leq 25^{\circ}$ and unsteady asymmetric flow with vortex shedding for $A O A \geq 30^{\circ}$. A number of passive control methods including vertical, fixed or erected, fins, two side strakes and four strakes in two different arrangements, oblique and cross, are used to restore the flow symmetry over the cone. A comparison between the studied control methods is carried out to select the best method of control. The four cross-strake method was found to be the best of the tested control methods.

\section{Keywords:}

Supersonic flow around conical bodies, high angle of attack, asymmetric flow around cones, passive control of asymmetric flow.

\footnotetext{
- Corresponding author e-mail address hamdykandil@hotmail.com

'Mechanical Engineering Department, Faculty of Engineering, Alexandria University, Alexandria, 21544, Egypt.
} 


\section{INTRODUCTION}

Slender conical shapes are among the most common shapes of the forebodies of supersonic missiles and aircraft fighters. Many research works have been dedicated to study the flow characteristics over such shapes both experimentally and computationally. The steady inviscid flow over these conical shapes is called conical flow which is a special case of three-dimensional flow where all flow quantities are invariant along rays that emanate from the cone apex. However, for unsteady viscous flow over a conical body, if two very close axial planes are considered, the flow may be thought of as "locally conical", with all flow quantities on these planes are invariant along rays that emanate from the cone apex, Liu, et. al. [1]. At high angles of attack, flow separations from the forebodies of missiles and fighter aircraft may become asymmetric resulting in side forces, yawing moments and rolling moments which are, in many instances, sufficiently large to trigger missiles and aircraft spin. Moreover, the resulting side forces can jeopardize the flight of aircrafts and decrease the targeting efficiency of self guided weapons, such as, missiles and intelligent bombs.

The research efforts to study the flow over conical bodies can be categorized into three historical stages. The first was the graphical solutions in 1929, Anderson [2]. The graphical solutions showed that the flow over circular cones at zero AOA is globally conical. When, the value of $\operatorname{AOA}(\alpha)$ is not zero the flow over slender conical bodies can be considered locally conical.

The second stage was the experimental studies which started by studying low-speed flows and then covered speeds up to hypersonic flows. The experimental studies [36 ] showed that as the AOA increases the crossing flow increases and forms a vortical flow over the conical bodies. Keener and Chapmann [7] categorized vortical-flow regimes into four distinct patterns depending on the AOA (Fig. 1). The first pattern develops in the very low AOA range, where the flow is attached and vortex free, and the axial flow is dominant. At moderate to high AOA, the cross-flow influence becomes of same order as that of the axial flow, and large scale vortices are formed on the leeward side of the body because of the boundary-layer separation (Fig. 2). At this $A O A$ range, the vortices are both stable and symmetric. At higher AOAs, the cross-flow effects start to dominate and the vortices may lose their stability or even symmetry, which may lead to asymmetric vortices about the symmetric body. The fourth flow pattern develops at extremely high AOAs where the cross-flow influences dominate completely, and leeside flow is characterized by an unsteady diffuse wake, which is the possibility of having either random or periodic vortex shedding. The previous researches showed that there are two mechanisms for explaining the evolution vortical-flow asymmetry over slender conical bodies at moderate and high AOAs. The first, suggests that the flow asymmetry occurs due to instabilities of velocity profiles in the vicinity of the saddle point that exists in the cross-flow planes above the projections of the body vortices, Fig. 2 . This mechanism appears to operate in both laminar and turbulent separation regimes. The second mechanism relates the asymmetry to the occurrence of asymmetric cross flow boundary-layer transition, leading to an effectively asymmetric mean flow about the body.

The third stage started in the 1990's after the great advancements in supercomputer which made many fiuid problems computationally solvable. Computational methods are advantageous over the experimental methods by their lower cost and higher flexibility to solve numerous cases without huge facilities or resources. 


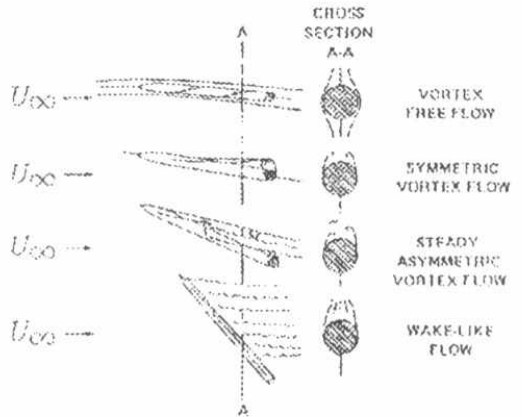

Fig. 1

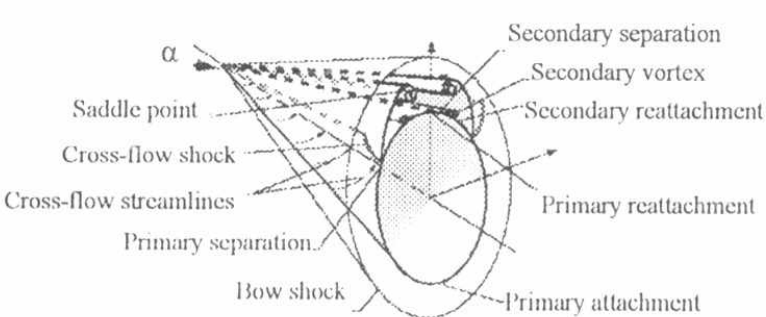

Fig. 2

The computational studies [ $8,9,10$ and11] simulate the flow asymmetry using two methods. The first is introducing a temporary imperfection in the boundary layers just for asymmetry initialization, which is known as convective instability. The second method depends on the random computational truncation errors to simulate the asymmetry initiator, which is known as absolute instability.

The great advancements in both experimental and computational devices encouraged many researchers to study control methods of flow asymmetry over slender bodies at moderate to high values of AOA. The control of this phenomena using passive or active control methods was studied both experimentally and computationally. In the field of computation, Kandil et. al. [12] and Malcolm [13] have studied using a vertical fin along the plane of geometric symmetry on the leeward side and side strakes as passive control methods. While different active methods such as jet blowing, suction, air injection, heating, spinning and rotary oscillation were studied by Font et. al. [14], Tavella et al. [15], and Kandil and Sharaf El-Din [16].

In the field of experimental work, same passive control methods were studied by Stahl [17] and Moskovitz [6]. Active control methods, air injection and jet blowing were studied by Skow and Peak [18] and Font et al. [14].

The present work covers the prediction of vortical flow asymmetry and vortex shedding over a $5^{\circ}$ semi-apex angle circular cone at a free-stream Mach number, $M_{\infty}=1.8$ and different $A O A s$, from $10^{\circ}$ to $30^{\circ}$. The flow is formulated using the full Navier-Stoke's equations and an implicit upwind flux-difference splitting finite-volume scheme is used to obtain the numerical solution of the governing equations. Also, a number of flow asymmetry control methods including vertical, fixed or erected fins, side strakes and four strakes in two different arrangements, oblique and cross, are examined. Then, a comparison between the studied control methods is presented.

\section{FORMULATION}

\section{Full Navier-Stokes Equations in General Coordinates:}

The governing equations for the compressible, viscous flow around the body are the conservative form of the dimensionless, unsteady compressible, full Navier-Stokes 
equations. Introducing the time-independent body-conforming coordinates, $\xi^{1}, \xi^{2}$ and $\xi^{3}$, yields the transformed equations as follows:

$$
\frac{\partial \hat{Q}}{\partial t}+\frac{\partial\left(\hat{E}_{m}-\hat{E}_{v_{m}}\right)}{\partial \xi^{m}}=0
$$

Where the flow-field vector, $\hat{\mathcal{C}}$, and the inviscid and viscous fluxes, $\hat{E}_{m}$ and $\hat{E}_{v m}$, are given by:

$$
\hat{Q}=\frac{Q}{J}=\frac{1}{J}\left[\begin{array}{c}
\rho \\
\rho \mathrm{u}_{1} \\
\rho \mathrm{u}_{2} \\
\rho \mathrm{u}_{3} \\
e_{t}
\end{array}\right], \hat{E}_{m}=\frac{\xi_{x_{n}}^{m} E_{n}}{J}=\frac{1}{J}\left[\begin{array}{c}
\rho \mathrm{U}_{\mathrm{m}} \\
\rho \mathrm{u}_{1} \mathrm{U}_{\mathrm{m}}+\xi_{x_{1}}^{m} p \\
\rho \mathrm{u}_{2} \mathrm{U}_{\mathrm{m}}+\xi_{x_{2}}^{m} p \\
\rho \mathrm{u}_{3} \mathrm{U}_{\mathrm{m}}+\xi_{x_{3}}^{m} p \\
\mathrm{U}_{\mathrm{m}}\left(e_{t}+p\right)
\end{array}\right] \text { and } \hat{E}_{v_{m}}=\frac{\xi_{x_{n}}^{m} E_{v_{n}}}{J}=\frac{1}{J}\left[\begin{array}{c}
0 \\
\xi_{x_{j}}^{m} \tau_{\mathrm{j}} \\
\xi_{x_{j}}^{m} \tau_{\mathrm{j} 2} \\
\xi_{x_{j}}^{m} \tau_{\mathrm{j} 3} \\
\xi_{x_{j}}^{m} \mathrm{~b}_{\mathrm{j}}
\end{array}\right]
$$

Where $m, n$ and $j$ are summation indices with values ranging from one to three. The Jacobian of transformation from the Cartesian coordinate to curvilinear space is given by:

$$
J^{-1}=\frac{1}{J}=\frac{\partial\left(x_{1}, x_{2}, x_{3}\right)}{\partial\left(\xi^{1}, \xi^{2}, \xi^{3}\right)}
$$

The contravarient velocity component in the direction $\xi^{m}$ is $U_{m}=\xi_{x_{j}}^{m} u_{j}$.

The shear stress and heat transfer elements in equation (2) are given by:

$\tau_{j l}=\frac{\mu M_{\infty}}{R_{\mathrm{e}}}\left(\frac{\partial \xi^{m}}{\partial x_{l}} \frac{\partial u_{j}}{\partial \xi^{m}}+\frac{\partial \xi^{m}}{\partial x_{j}} \frac{\partial u_{l}}{\partial \xi^{m}}-\frac{2}{3} \delta_{j l} \frac{\partial \xi^{m}}{\partial x_{k}} \frac{\partial u_{k}}{\partial \xi^{m}}\right)$

and $b_{j}=u_{k} \tau_{j k}+\frac{\mu M_{\infty}}{(\gamma-1) P_{r} R_{e}} \frac{\partial \xi^{m}}{\partial x_{j}} \frac{\partial a^{2}}{\partial \xi^{m}}$, respectively.

Where, $j$ and $/$ are free indices and $k$ and $m$ are summation indices. The range of $j, k$, $l$ and $m$ is from one to three. The reference parameters for the dimensionless form of the equations are $L, a_{\infty}, L / a_{\infty}, \rho_{\infty}$ and $\mu_{\infty}$ for the length, velocity, time, density and molecular viscosity, respectively. The freestream Reynolds number is defined as $\operatorname{Re}$ $=\rho_{\infty} V_{\infty} L / \mu_{\infty}$, where $L$ is the body length, $a_{\infty}, \rho_{\infty}$ and $\mu_{\infty}$ are the free-stream speed of sound, density and viscosity, respectively.

The pressure, $p$, is related to the total energy per unit mass for an ideal gas equation by the following equation: $p=(\gamma-1) \rho\left[c-\frac{1}{2}\left(u_{1}^{2}+u_{2}^{2}+u_{3}^{2}\right)\right]$.

The viscosity is calculated from the Sutherland's law, $\mu=T^{3 / 2}\left(\frac{1+C}{T+C}\right), \mathrm{C}=110.4 \mathrm{~K}$. The Prandtl number Pr is chosen to be 0.72 .

\section{Computational Method:}

Equation (1) is written in the integral form and the divergence theorem is applied to the divergence of the inviscid and viscous flux terms. 


$$
\begin{aligned}
& \iiint_{R} \frac{\partial \hat{Q}}{\partial t} d v+\iint_{\partial R}\left(\hat{E}_{1}-\hat{E}_{v_{1}}\right) d \xi^{2} d \xi^{3}+\iint_{\partial R}\left(\hat{E}_{2}-\hat{E}_{v_{2}}\right) d \xi^{1} d \xi^{3} \\
& +\iint_{\partial R}\left(\hat{E}_{3}-\hat{E}_{v_{3}}\right) d \xi^{1} d \xi^{2}=0
\end{aligned}
$$

Where, $R$ is the domain of integration and $\partial R$ is the domain's boundary. The computational domain, $\mathrm{R}$ is divided into small hexahedral cells which are considered as unit cubes, for convenience. The flow quantities are cell-averaged values rather than point wise values at the cell center. The integral form of Eq. (3) becomes an ordinary differential equation in time with spatial-differencing terms.

$$
\begin{aligned}
J_{i, j, k}^{1}\left(\frac{\partial Q}{\partial t}\right)_{i, j, k}= & {\left[\left(\hat{E}_{1}-\hat{E}_{v_{1}}\right)_{i+\frac{1}{2} j, k}-\left(\hat{E}_{1}-\widehat{E}_{v_{1}}\right)_{i-\frac{1}{2} j, k}\right.} \\
& +\left(\widehat{E}_{2}-\widehat{E}_{v_{2}}\right)_{i, j+\frac{1}{2}, k}-\left(\widehat{E}_{2}-\widehat{E}_{v_{2}}\right)_{i,-\frac{1}{2}, k} \\
& \left.+\left(\widehat{E}_{3}-\widehat{E}_{v 3}\right)_{i, j, \frac{1}{2}}-\left(\widehat{E}_{3}-\widehat{E}_{v_{3}}\right)_{i j, k-\frac{1}{2}}\right]=R\left(\hat{Q}_{i, j, k}\right)
\end{aligned}
$$

Where, the subscripts $i, j, k$ denote the value at the centroid of a cell and the halfinteger subscripts refer to the value at the interface of cells.

Then the flux-difference splitting scheme of Roe is applied on the equations. The Jacobian matrices of the inviscid fluxes are split into backward and forward fluxes according to the sign of the eigen values of the inviscid Jacobian matrices. The viscous and heat-flux terms are centrally differenced. The resulting difference equation is solved using approximate factorization in the $\xi^{1}, \xi^{2}$ and $\xi^{3}$ directions. Locally-conical solutions of the Navier-Stokes equations are obtained by solving the three-dimensional flow equations on two axial (or cross-flow) surfaces which are located in the very near proximity of a constant axial distance. During time stepping, the flow field vector is forced to be equal at the corresponding grid cell centers on the two surfaces.

\section{Initial and Boundary Conditions:}

The initial conditions correspond to uniform flow with $u_{1}=u_{2}=u_{3}=0$ on the solid boundary. On the solid boundary, the no-slip and no-penetration conditions are enforced, i.e., $u_{1}=u_{2}=u_{3}=0$ and the normal pressure gradient is assumed to be zero. The adiabatic condition is maintained on the solid surfaces. In the plane of geometric symmetry, periodic conditions are used. Far field boundary conditions are specified to be freestream conditions. Solid boundary conditions are applied on both sides of fins and strakes.

\section{PREDICTION OF FLOW ASYMMETRY OVER A CIRCULAR CONE}

Navier-Stokes equations are used to solve for asymmetric flow on a grid of $161 \times 81$ points in the circumferential and normal directions, respectively. The global time step is $10^{-4}$ is used in all case studies. The grid was generated using a modified Joukowski transformation and a geometric series was used for grid clustering in both circumferential and normal directions. The minimum grid spacing in both directions is $2 \times 10^{-3}$. The maximum radius of the computational domain is $15 r_{b}$, where $r_{b}$ is the radius of the circular cone at the axial station of unity. 
Steady Asymmetric Flows over a Circular Cone $\left(15^{\circ} \leq \alpha \leq 25^{\circ}, M_{\infty}=1.8, R e=10^{5}\right)$

Figures 3.a, 3.b, 3.c and 3.d show the results of studying the flow over a $5^{\circ}$ semiapex circular cone at $M_{\infty}=1.8$ and $R e=10^{5}$ and $A O A(\alpha)$ equals $15^{\circ}, 20^{\circ}$ and $25^{\circ}$ Figure 3.a shows total pressure loss contours over the cone for the three cases. Figure 3.b shows the history of residual error while Fig. 3.c shows the pressure coefficients versus body angle, theta. Figure 3.d shows the history of the side force coefficients.

The results of the three cases represent steady asymmetric vortical flows. The total pressure loss contours show that, as the AOA increases the asymmetry of the two vortices over the leeward side of the slender cone increases. Results of the residual error show that the numbers of time steps required to obtain steady state at $\alpha=15^{\circ}$, $20^{\circ}$ and $25^{\circ}$ are 800,2000 and 3400 , respectively. This means that as the AOA increases the time required for the asymmetric vortical flow to become steady increases. The surface pressure coefficient distributions show that there is an asymmetry of the surface pressure coefficient on the two sides of the cone. This asymmetry increases as the AOA increases. The asymmetry in surface pressure causes a steady side force which is shown Fig. 3.c. Figure 3.c shows that the side force increases as the AOA increases. The vortices demonstrated by the total pressure loss contours in Fig. 3.a are steady asymmetric at these values of AOA. The results show that as the AOA increases the size of the vortices increases and the vortices become weaker. The weakness of vortices structure explains their need for more time to reach steadiness. The results in the next section will show that at higher AOAs the vortices will be too weak to attain steadiness.

Unsteady Asymmetric Flow with Vortex Shedding $\left(\alpha=30^{\circ}, M_{\infty}=1.8, \operatorname{Re}=10^{5}\right.$ )

As the AOA increases the leeward side vortices become larger and weaker. The weakness of the vortices makes it difficult to reach a steady state. At an angle of attack, $\alpha=30^{\circ}$, the flow was found to be unsteady asymmetric with vortex shedding. Eight snapshots of the total pressure-loss contours after 7700 time steps with an increment of 200 time steps are shown in Fig. 4. The figure shows a clear demonstration of an unsteady flow asymmetry with clear vortex shedding from both sides. The shedding mechanism occurs in a periodic manner. It takes about 1200 time steps to complete one cycle of vortex shedding oscillation. It is noticed that the second snapshot (at $n=7900$ ) is very similar to the last snap shot shown (at $n=$ 9100 ) while the snapshot (at $n=8500$, the mid cycle) is a mirror image to the two snapshots mentioned above. As a result of the periodic vortex shedding, the conical body oscillates which may jeopardize the flight safety.

\section{PASSIVE CONTROL OF SUPERSONIC ASYMMETRIC VORTICAL FLOWS AROUND CONES}

\section{Flow Asymmetry Control Using Vertical Fins:}

Vertical fins in the leeward plane of geometric symmetry were examined experimentally by Stahl [17] and computationally by Kandil et al. [12] in controlling the flow asymmetry around cones at $A O A=20^{\circ}, M_{\infty}=1.8$ and $\mathrm{Re}=10^{5}$. Their results showed that the presence of a vertical fin suppressed the vortical flow 
asymmetry. The minimum fin height to control the asymmetry was found to be equal to the local radius of the cone. However, both the numerical and experimental studies tested only two different fin heights $\left(0.5 r_{b}\right.$ and $\left.1 r_{b}\right)$ at $A O A=20^{\circ}$. The margin from $0.5 r_{b}$ to $1 r_{b}$ is a wide margin that led to bad resolution. In the present, a numerical simulation of the vortical flow using vertical fins with heights from $0.1 r_{b}$ to $1.35 r_{b}$ shows that at $\operatorname{AOA}(\alpha)=20^{\circ}$ a fin with a height less than $1 r_{b}$ is capable in eliminating the flow asymmetry. Also, using an erected fin is examined at the same $\mathrm{AOA} \alpha=20^{\circ}$.

This section considers the use of vertical fins to control the flow asymmetry at AOAs of $20^{\circ}$ and $30^{\circ}$, as they represent the two conditions of flow asymmetry; steady asymmetric vortical flow and unsteady asymmetric vortical flow with vortex shedding.

\section{Using Fixed Vertical Fins to Control The Flow Asymmetry:}

In this method the fin is placed from the beginning of the computational simulation. This situation represents the case in which the fin is fixed to the body. Figures 5.a and 5.b show the results of using vertical fixed fins of heights of $0.4 r_{b}$ and $0.6 r_{b}$ at $\alpha$ $=20^{\circ}$, while figures 5.c and 6.d show the results when using fixed fins of heights of $0.8 \mathrm{r}_{\mathrm{b}}$ and $1.25 \mathrm{r}_{\mathrm{b}}$ at $\alpha=30^{\circ}$. It is noticed that using a vertical fin of a height of $0.6 \mathrm{r}_{\mathrm{b}}$ is enough to maintain the flow symmetry at $\alpha=20^{\circ}$. This height is less than the minimum height required at same AOA in reference [12]. Figure 5.d shows that the minimum required height to maintain the flow symmetry at $\alpha=30^{\circ}$ is $1.25 \mathrm{r}_{\mathrm{b}}$ which is very large and also not practical.

\section{Using Vertical Erected Fins to Control the Flow Asymmetry:}

In this method the computational simulations start without the presence of the vertical fin then the fin is placed gradually (erected) in the plane of geometric symmetry if a flow asymmetry is sensed. This situation simulates a case in which a fin folded inside the body is erected only if a flow asymmetry is sensed over the leeward side of the body. This control method may be classified as an active control method since the method is used only if it is needed and the height of the fin may be adjusted based on the strength of the vortex asymmetry. The erection can be controlled by a hydraulic cylinder with a proportional control system that senses the pressure difference between the two sides of the cone. This flow control method was never simulated in the literature and further investigation is required to judge its visibility and applicability. Figures $6 . a$ and $6 . b$ show the results of using erected fins of heights of $0.6 \mathrm{r}_{\mathrm{b}}$ and $0.75 \mathrm{r}_{\mathrm{b}}$ at $\alpha=20^{\circ}$, while Figs. 6.c and 6.d show the results of using erected fins of heights of $1.25 \mathrm{r}_{\mathrm{b}}$ and $1.35 \mathrm{r}_{\mathrm{b}}$ at $\alpha=30^{\circ}$. It is noticed that using an erected vertical fin of a height of $0.6 \mathrm{r}_{\mathrm{b}}$ is not enough to maintain the flow symmetry at $\alpha=20^{\circ}$. Although this height was enough when using a fixed fin. Figure $6 . b$ shows that the minimum erected fin height required restore the flow symmetry is $0.75 r_{b}$. Figures $6 . c$ and $6 . d$ show that the minimum required fin height restore the flow symmetry at $\alpha=30^{\circ}$ is $1.35 r_{b}$ which is very large and seems to be impractical.

\section{Control of Flow Asymmetry Using Two and Four Thick Strakes:}

Different arrangements and lengths of strakes are used to control vortical flow asymmetry at AOA values of $20^{\circ}, 30^{\circ}$ and $35^{\circ}$. Flow conditions and strake's slope angles will be the same for all considered cases $\left(M_{\infty}=1.8, \operatorname{Re}=10^{5}, \delta=30^{\circ}\right)$. 


\section{Using Two Sharp Edged Thick Side Strakes to Control the Flow Asymmetry}

Figures 7 and 8 show the total pressure-loss contours, residual error and surface pressure coefficient when using two $0.3-r_{b}$-length side strakes at AOAs of $20^{\circ}$ and $30^{\circ}$. The results show that the side strakes render the flow perfectly symmetric since the two primary vortex cores are pushed further apart preventing the flow disturbances of the two sides from interacting. The effects of placing the side strakes on the surface pressure distribution is very clear from Figs. 7 and 8 which has the favorable effect of increasing the lift coefficient as was also presented by Liu et. al. [19].

The results of using strakes of the same length of $0.3 r_{b}$ at $\alpha=35^{\circ}$ are shown in Figs. 9. a and 10.b. The figure shows that at $\alpha=35^{\circ}, 0.3-r_{b}$-length strakes are not enough to maintain the flow symmetry. The flow in this case is unsteady with vortex shedding. Testing larger strakes at the same AOA showed that the minimum required strake length to maintain the flow symmetry is $0.8 r_{b}$. This result is shown in Fig. 9.c, which shows the total pressure-loss contours, residual error versus number of time steps and surface pressure coefficient versus body angle for using two side strakes of $0.8 r_{b}$ length at $\alpha=35^{\circ}$.

\section{Using Four Oblique Strakes and Four Cross Strakes to Control the Flow}

\section{Asymmetry}

Two different arrangements of four strakes on a slender cone are tested to control the vortical flow asymmetry occurring at AOAs of $20^{\circ}, 30^{\circ}$ and $35^{\circ}$ at the same flow conditions of the previous cases. It is noted that no computational or experimental results of using this control methods were presented by previous investigators in the literature. The first arrangement consists of four oblique strakes placed at angles of $45^{\circ}$ from the plane of geometric symmetry, while the second arrangement consists of four cross strakes. Figures 10.a and 10.b show the total pressure-loss contours and surface pressure coefficient of the flow around a cone at $\alpha=20^{\circ}$ and $\alpha=35^{\circ}$. The results show that the minimum strake lengths required to maintain the flow symmetry at these values of AOA are $0.3 r_{b}$ and $0.6 r_{b}$, respectively. Figures 10.c and 10.d show the total pressure-loss contours and surface pressure coefficient at $\alpha=20^{\circ}$ and $\alpha=35^{\circ}$ for using four cross strakes. The results show that the minimum strake lengths of the four cross strakes required to keep the flow symmetry at $\alpha=20^{\circ}$ and $\alpha$ $=35^{\circ}$ are $0.3 r_{b}$ and $0.6 r_{b}$, respectively.

\section{Comparison between Different Control Methods}

The results presented in the previous sections show that, in all control methods the higher the AOA the longer length of control device is required. It is noticed that the required length of the vertical fin is the highest among all the control methods. Also, using an erected fin instead of fixed fin requires the fin to be larger. The length of two side strakes to maintain the flow symmetry at $\alpha=35^{\circ}$ was found to be $1 r_{b}$ while, the required length of the four oblique or cross strakes for the same AOA is $0.6 r_{b}$. Although, the required length of both oblique and cross strakes are the same, the surface pressure distributions, shown in Fig. 10, show that the flow stability and lift 
forces are better in case of using four cross strakes than in case of using four oblique strakes.

\section{CONCLUSIONS}

- The results show that the flow asymmetry around conical bodies occurs when the angle of attack exceeds certain critical values. For angle of attack range: $15^{\circ} \leq \alpha \leq 25^{\circ}$, the vortical flow was found to be steady asymmetric. At higher values of $A O A, \alpha \geq 30^{\circ}$, the flow becomes unsteady asymmetric with periodic vortex shedding.

- Vertical thin fins, either fixed from the beginning of simulation or erected after the development of flow asymmetry, along the plane of symmetry in the leeward side of the cone were successful in controlling the flow asymmetry.

- Erecting the fin after the development of flow asymmetry required higher length of the fin than that required when for a fixed fin.

- Fin lengths required to control the vortical flow at $\alpha \geq 30^{\circ}$ were too large to be practical.

- Two side strakes with lengths less than that of the corresponding vertical fins at the same flow conditions were capable of controlling the flow at AOA values up to $35^{\circ}$. The required strake length at $A O A$ of $35^{\circ}$ is $0.8 r_{b}$.

- Four strakes in two different arrangements, cross and oblique, were used to control the asymmetric flow at $A O A$ values up to $35^{\circ}$. The required strake length to control the flow at $\alpha=35^{\circ}$ was found to be $06 r_{b}$.

- The four cross-strake method was found to be the best of the tested control methods.

\section{REFERENCES}

1. Liu, C.H., Wong, T-C. and Kandil, O.A. ,"Prediction of Asymmetric Vertical Flows Around Slender Bodies Using Navier-Stokes Equations," Fluid Dynamics Research, Vol. 10, pp. 409-450, 1992.

2. Anderson, J. D., Jr., "Modern Compressible Flow with historical prospective," McGRAW-HILL, ISBN 0-07-100665-6, 1982.

3. Lamont, P.J.," The Complex Asymmetric Flow over a 3.5d Ogive Nose and Cylindrical Afterbody at High Angles of Attack," AIAA paper 82-0053, 1982.

4. Lamont, P.J.," Pressure Measurements on an Ogive-Cylinder at High Angles of Attack with Laminar, Transition or Turbulent Separation," AIAA paper 80-1556cp, 1980.

5. Degani, D. and Zilliac, G. G., "Experimental Study of Unsteadiness of the Flow around an Ogive-Cylinder at Incidence," AIAA paper 88-4330, 1988.

6. Moscovitz, C., Hall, R. and Dejarnette, "Experimental Investigation of a New Device to Control the Asymmetric Flowfield on Forebodies at Large Angle of Attack," AIAA 90-0069, 1990.

7. Keener, E.R. and Chapman, G.T., "Similarity in Vortex Asymmetries over Slender Bodies and Wings," AIAA Journal, vol. 15, pp. 1370-1372, Sept. 1977. 
8. Godavarty, D. and Orkwis, P. D., "Investigating the Relationship between Flow Field Saddles and Vortex Asymmetry, " AIAA paper 2001-1034, 2001.

9. Orkwis, P., Sengupta, R. and Davis, S.M., "Flow Field Saddles and their Relation to Vortex Asymmetry," Computers and Fluids, 26, 505, 1997.

10. Kandil, O.A., Wong, T-C. and Liu, C.H.," Prediction of Steady and Unsteady Asymmetrical Vortical Flow around Cones," AIAA paper 90-0598, January 1990.

11. Kandil, O.A., Wong, T-C. and Liu, C.H., "Asymmetric Flow around Cones with Noncircular Sections," AGARD Symposium on Missile Aerodynamics, paper No.16, Friedrichshafen, FRG, April 1990.

12. Kandil, O.A., Wong, T-C. and Kandil, H. A., " Computation and Control of Asymmetric Vortex Flow around Circular Cones using Navier-Stokes Equation," ICAS paper No. 3.5.3., Vol. 2 ,Stockholm, Sweden, pp. 383-893, September 1990.

13. Malcolm GN. "Forebody Vortex Control a Progress Review " AIAA paper 933540, 1993.

14. Font, G. I., Celik, Z. Z. and Roberts, L., "A Numerical and Experimental Study of Tangential Jet Blowing Applied to Bodies at High Angles of Attack," Proceedings of the AIAA $9^{\text {th }}$ Applied Aerodynamics Conference, Baltimore, Maryland, AIAA paper 91-3253-CP, pp. 400-411, 1991.

15. Tavella, D. A., Schiff, L. B. and Cummings, R. M., "Pneumatic Vortical Flow Control at High Angles of Attack," AIAA paper 90-0098, 1990.

16. Kandil, O. A., and Sharaf El-Din, H. H., "Active Control of Asymmetric Conical Flow Using Spinning and Rotary Oscillation," AIAA paper 93-2958, July 1993.

17. Stahl, W., "Suppression of Asymmetry of Vortex Flow Behind a Circular cone at High Incidence," AIAA paper 89-3372-CP, Aug. 1989.

18. Skow, A. M. and Peake, D. J., "Control of the Forebody Vortex Orientation by Asymmetric Air Injection, (Part B) - Details of the Flow Structure, " AGARD-LS121, High Angle of Attack Aerodynamics, pp.10.1-10.22, 1982.

19. Liu, C.H., Kandil, O.A. and Wong, T-C., "Passive Control of Supersonic Asymmetric Vortical Flows around Cones," Impact of Computing in Science and Engineering, Vol. 4, pp. 80-96, 1992. 


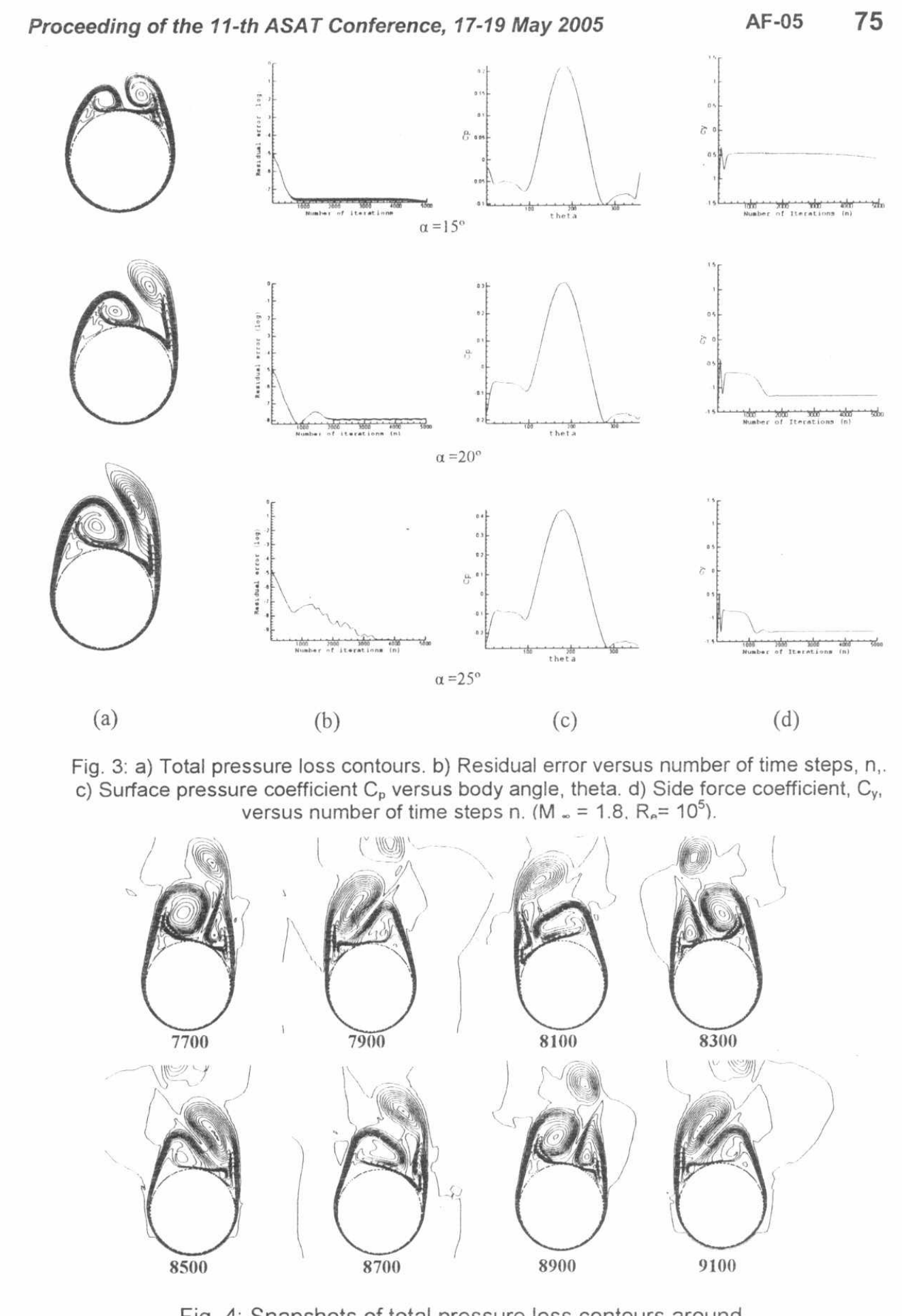


Proceeding of the 11-th ASAT Conference, 17-19 May 2005
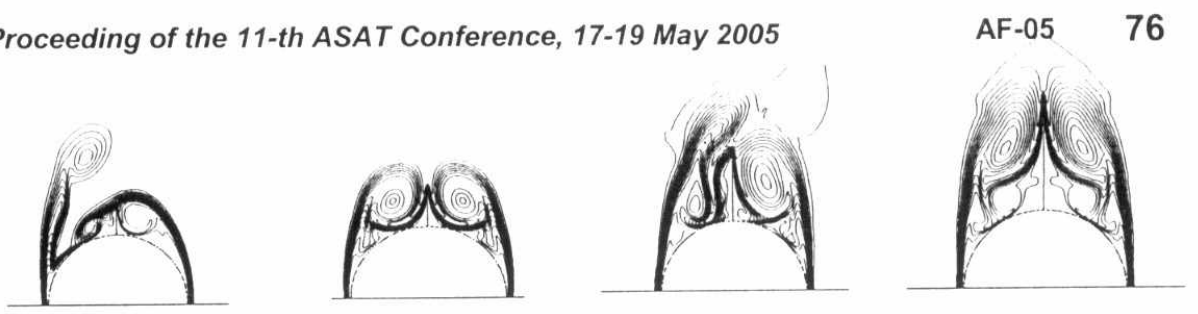

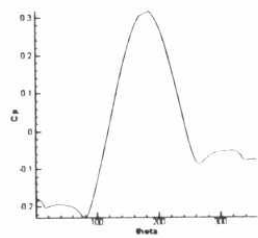

(a)

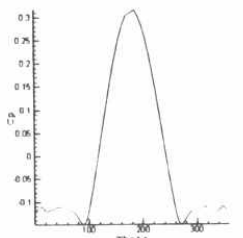

(b)

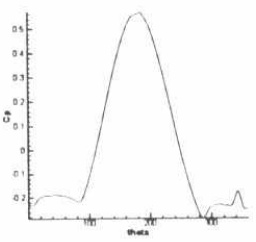

(c)

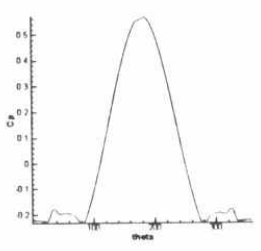

(d)

Fig. 5: Total pressure-loss contours and surface pressure coefficient distribution versus body angle theta at $\alpha=20^{\circ}$ and using fixed fin with lengths a) $0.4 r_{b}$. b) $0.6 r_{b}$ and at $\alpha .=30^{\circ}$ and using fixed fin with length c) $0.8 r_{b}$. d) $1.25 r_{b}$
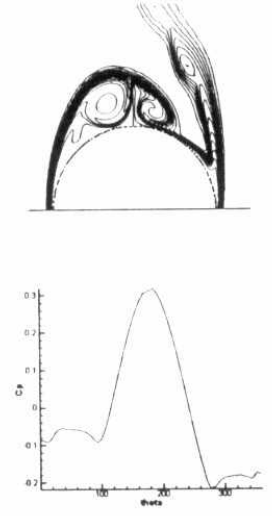

(a)
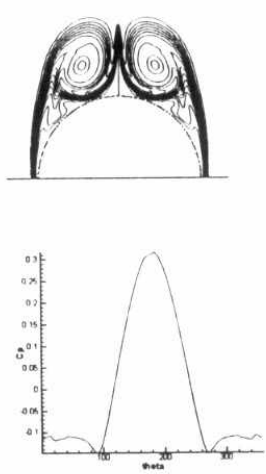

(b)
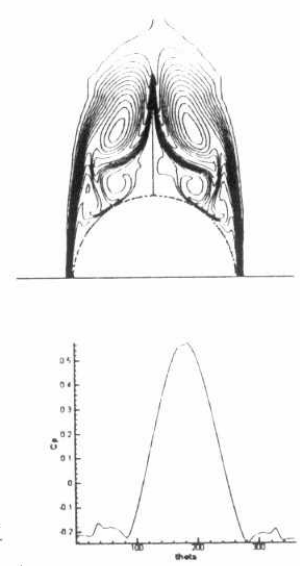

(c)
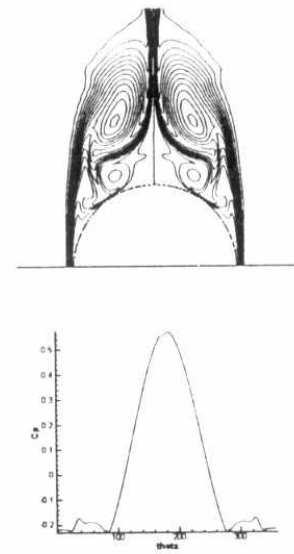

(d)

Fig. 6: Total pressure-loss contours and surface pressure coefficient distribution versus body angle theta at $\alpha .=20^{\circ}$ and using erected fins with lengths a) $0.6 r_{b}$. b) $0.75 r_{b}$ and at $\alpha=30^{\circ}$ and using erected fins with lengths c) $1.25 \mathrm{r}_{\mathrm{b}}$. d) $1.35 \mathrm{r}_{\mathrm{b}}$

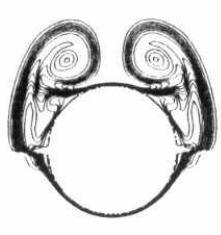

(a)

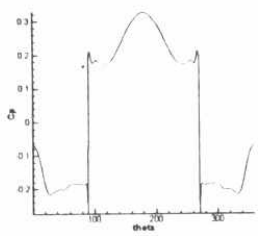

(b)

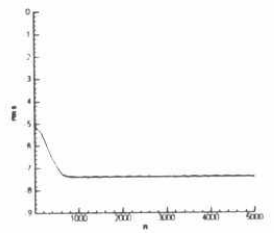

(c)

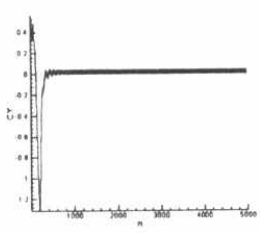

(d)

Fig. 7: a) Total pressure loss contours. b) Surface pressure coefficient $C_{p}$ versus body angle theta. c) Residual error versus number of time steps. d) Side force coefficient $\mathrm{C}_{\mathrm{y}}$ versus time steps At $\alpha=20^{\circ}$ when using 0.3- $r_{b}$-length side strakes. 


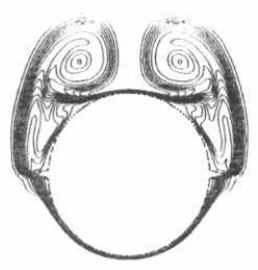

(a)

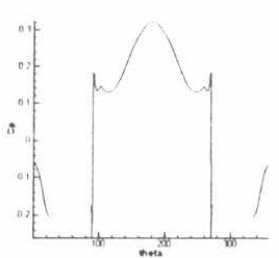

(b)

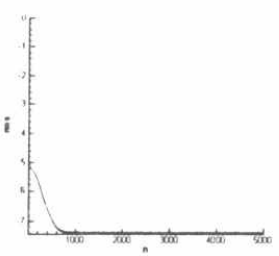

(c)

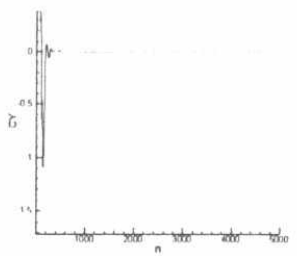

(d)

Fig. 8: a) Total pressure loss contours. b) Surface pressure coefficient $C_{p}$ versus body angle theta. c) Residual error versus number of time steps $n$. d) Side force coefficient $\mathrm{C}_{\mathrm{y}}$ versus number of time steps. At $\alpha=30^{\circ}$ when using two $0.3-r_{b}$-length side strakes.
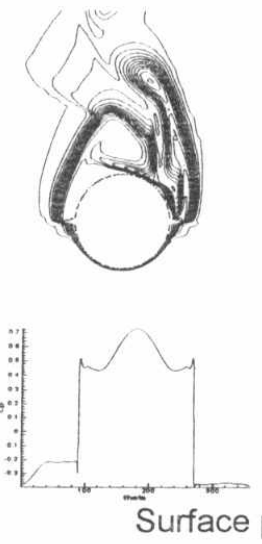

(a)

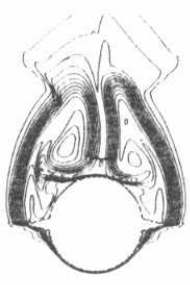

Total pressure loss contours

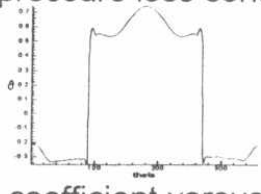

(b)
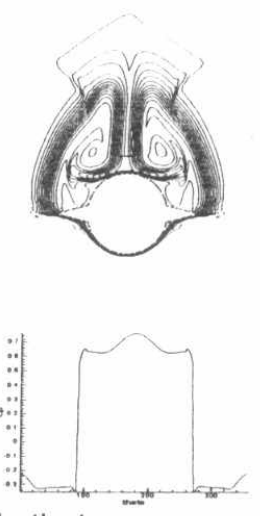

(c)

Fig. 9: Total pressure loss contours and surface pressure coefficient versus body angle theta at $\alpha=35^{\circ}$ using a) $0.3-r_{b}$-length two side strakes. b) $0.5-r_{b}$-length two side
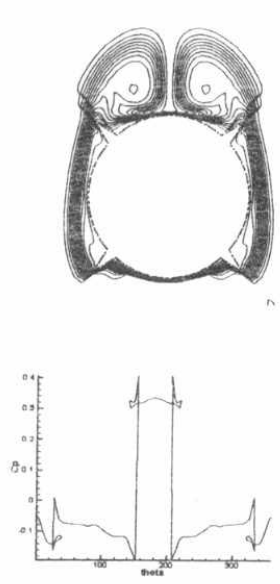

(a)
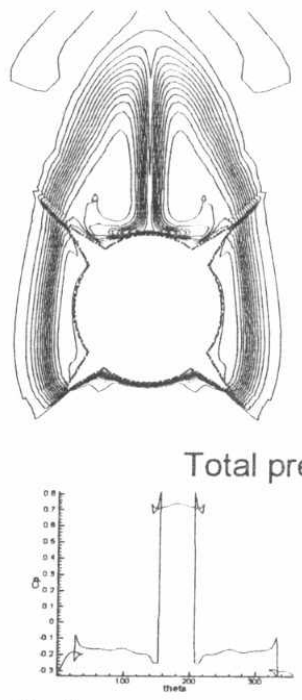

Surface press
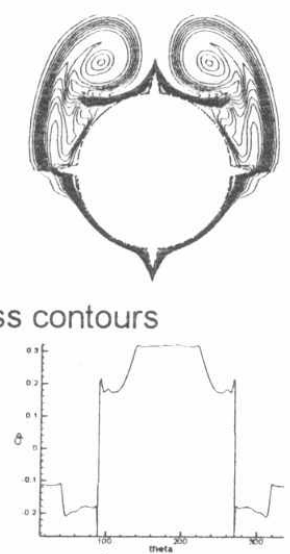

(c)
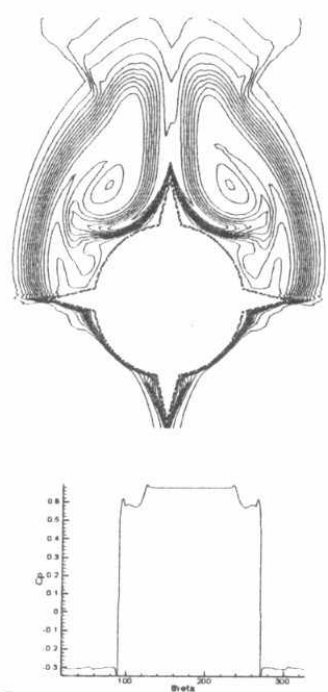

(d)

Fig. 10: Total pressure-loss contours and surface pressure coefficient at a) $\alpha=20^{\circ}$ using $0.3-r_{b^{-}}$ length four oblique strakes. b) $\alpha .=35^{\circ}$ using $0.6-r_{b}$-length four oblique strakes. c) $\alpha=20^{\circ}$ using 0.3- $r_{b}$-length four cross strakes. d) $\alpha=35^{\circ}$ using 0.6- $r_{b}$-length four cross strakes. 


\section{HYDRAULICS \& FLUID SYSTEMS}




\section{CONTENTS}

FH-01 COMPUTATIONAL AND EXPERIMENTAL INVESTIGATION

OF OPEN CAVITIES FLOW

N. N. Bayomi, A. M. Abdel Dayem

FH-02 COMPUTATIONAL SIMULATION AND EXPERIMENTAL

VALIDATION OF DOUBLE SQUARE CYLINDERS FLOW IN STAGGERED ARRANGEMENT

A. M. Abdel Dayem and N. N. Bayomi

FH-03 EXPERIMENTAL AND THEORETICAL INVESTIGATION OF SUSPENSION

Z. A. Ibrahim, M.G. Rabie, S.A. Hegazy, I.A. El-Sherif

FH-04 DESIGN AND ANALYSIS OF DYNAMIC PERFORMANCE OF A VEHICLE ACTIVE SUSPENSION SYSTEM

Z. A. Ibrahim , I.A. El-Sherif, M.G. Rabie, S.A. Hegazy

FH-05 SUPPRESSION OF VORTEX INDUCED VIBRATIONS ON MARINE RISERS VIA BOUNDARY LAYER SUCTION

Farouk M. Owis

FH-06 DETERMINATION OF OPTIMUM SLOT IN A BACKWARD VANE IMPELLER FOR BETTER CENTRIFUGL PUMP PERFORMANCE Hussien M.S.E, Lotfy A.H., Abdalla H.M., and Saleh I.

FH-07 PERFORMANCE OF OIL-INJECTED TWIN-ROTORS SCREW COMPRESSOR

Kassab S. Z., Adam I. G. , El-Gohary H. A.

FH-08 EXPERIMENTAL INVESTIGATION ON THE INFLUENCE OF MUZZLE BRAKE GEOMETRY ON AUTOMATIC SMALL ARMS PERFORMANCE

Moghazy, M. A.

FH-09 EXPERIMENTAL INVESTIGATION ON CONTROLLING OF MUZZLE BLAST WAVE IN AUTOMATIC SMALL ARMS

Moghazy, M. A. 